\title{
The Danish National Chronic Myeloid Neoplasia Registry
}

This article was published in the following Dove Press journal:

Clinical Epidemiology

25 October 2016

Number of times this article has been viewed

\author{
Marie Bak' \\ Else Helene Ibfelt ${ }^{2}$ \\ Thomas Stauffer Larsen ${ }^{3}$ \\ Dorthe Rønnov-Jessen ${ }^{4}$ \\ Niels Pallisgaard ${ }^{5}$ \\ Ann Madelung ${ }^{6}$ \\ Lene Udby' \\ Hans Carl Hasselbalch' \\ Ole Weis Bjerrum ${ }^{7}$ \\ Christen Lykkegaard \\ Andersen ${ }^{1,7}$ \\ 'Department of Hematology, Zealand \\ University Hospital, University of \\ Copenhagen, Roskilde, ${ }^{2}$ Research \\ Centre for Prevention and Health, \\ Rigshospitalet Glostrup, University of \\ Copenhagen, Glostrup, ${ }^{3}$ Department \\ of Hematology, Odense University \\ Hospital, Odense, ${ }^{4}$ Department of \\ Hematology, Vejle Hospital, Vejle, \\ ${ }^{5}$ Department of Surgical Pathology, \\ Zealand University Hospital, \\ University of Copenhagen, Roskilde, \\ ${ }^{6}$ Department of Surgical Pathology, \\ Zealand University Hospital, \\ University of Copenhagen, Næstved, \\ ${ }^{7}$ Department of Hematology, \\ Rigshospitalet, University of \\ Copenhagen, Copenhagen, Denmark
}

Correspondence: Marie Bak

Department of Hematology, Zealand

University Hospital, University of

Copenhagen, Sygehusvej 10,4000

Roskilde, Denmark

Tel +45 47324894

Fax +4547324890

Email doctormariebak@gmail.com
Aim: The Danish National Chronic Myeloid Neoplasia Registry (DCMR) is a population-based clinical quality database, introduced to evaluate diagnosis and treatment of patients with chronic myeloid malignancies. The aim is to monitor the clinical quality at the national, regional, and hospital departmental levels and serve as a platform for research.

Study population: The DCMR has nationwide coverage and contains information on patients diagnosed at hematology departments from January 2010 onward, including patients with essential thrombocythemia, polycythemia vera, myelofibrosis, unclassifiable myeloproliferative neoplasms, chronic myelomonocytic leukemia, and chronic myeloid leukemia.

Main variables: Data are collected using standardized registration forms (so far up to four forms per patient), which are consecutively filled out online at time of diagnosis, after 2-year and 5-year follow-ups, and at end of follow-up. The forms include variables that describe clinical/ paraclinical assessments, treatment, disease progression, and survival - disease-specific variables - as well as variables that are identical for all chronic myeloid malignancies.

Descriptive data: By the end of 2014, the DCMR contained data on 2,690 patients with an inclusion rate of $\sim 500$ patients each year. Since the registry was established, annual reports have shown consistently high national coverage and data completeness, $\geq 90 \%$ and $\geq 88 \%$, respectively.

Conclusion: The DCMR is a national database used for monitoring the quality of patient care in patients with chronic myeloid malignancies, but until validation has been conducted, the data must be used with caution. However, the DCMR is a valuable data source accessible to clinicians and researchers.

Keywords: myeloproliferative disorders, database, treatment, health care quality assurance, outcome assessment, epidemiology, research

\section{Introduction}

The Danish National Chronic Myeloid Neoplasia Registry (DCMR) was established on January 1, 2010, to monitor the clinical quality of diagnosis and treatment in patients with chronic myeloid malignancies. This population-based clinical quality database with nationwide coverage contains information on patients with essential thrombocythemia, polycythemia vera, myelofibrosis, unclassifiable myeloproliferative neoplasms, chronic myelomonocytic leukemia, and chronic myeloid leukemia. The DCMR is part of the Danish Common Hematological Database established by the Danish Society of Hematology, to evaluate patients with various hematological malignancies and to ensure the quality of diagnostic evaluation, treatment, and patient care at the national level, as well as in each hematology department. 


\section{Aim of the database}

The aim of the DCMR is to collect information on patients with different subtypes of chronic myeloid malignancies, to gain insight into disease epidemiology, and to characterize patients by clinical and paraclinical evaluation at predefined intervals throughout the course of the cancers. The DCMR enables nationwide monitoring of the quality of diagnosis and treatment in patients with chronic myeloid malignancies in Denmark. As with all government-approved clinical quality databases, a continuous aim is also to ensure coverage $>90 \%$ and registration completeness $>80 \%$ each year, at the national, regional, and hospital departmental levels. The database can also be used to compare different patientand treatment-related outcomes between departments. The DCMR serves as a quality database as well as a research database.

\section{Study population}

Danish citizens with chronic myeloid malignancies, referred to hematology departments for evaluation since January 1, 2010, are eligible for registration in the DCMR. All nine hematology departments in Denmark are obliged to collect and enter data. Patients diagnosed or treated outside Denmark are included only if their treatment is continued in Denmark. The patients must fulfill the current World Health Organization classification criteria for essential thrombocythemia, polycythemia vera, myelofibrosis, unclassifiable myeloproliferative neoplasms, chronic myelomonocytic leukemia, or chronic myeloid leukemia to be included in the DCMR. ${ }^{1}$

The Danish health care system provides free health care to all 5.5 million inhabitants, ${ }^{2}$ which enables nationwide coverage of the DCMR. In 2009, the Danish Health and Medicines Authority introduced the hematological cancer care packages, as part of the national initiative termed The National Integrated Cancer Pathways. ${ }^{3}$ The regulation included guidelines for Danish patients with hematological cancer, implying that all patients with a suspected hematological cancer must be evaluated at a hematology department. This ensures that data on virtually all in- and outpatients with chronic myeloid malignancies are included in the DCMR, and data are collected on $\sim 500$ new patients each year.

\section{Registration system and variables Registration system}

The Web-based registration system for entering data into the DCMR (as well as all other hematological registries) is based on a reporting tool, developed using SAS Institute software version 9.2 (SAS Institute Inc, Cary, NC, USA). The entered data are securely stored electronically. The online registration system is easily accessible from every department in Denmark, and physicians do not need patients' consent when entering data. Data are collected from patients' pathology and laboratory reports, medical files, and assessments by the consulting hematologists. Trained health care professionals enter data on all eligible patients into the system manually, using standardized registration forms. Instructions for entering data are available online to ensure a homogeneous national registration. Validation rules, including field and cross-validation, restrict what can be entered, and each variable must be answered before the registration forms can be submitted, thus optimizing the completeness of the registration.

\section{Registration forms and main variables}

For each patient, data collected on up to four registration forms entered at predefined intervals are included in the DCMR so far. The registration algorithm for the DCMR is shown in Figure 1. The different forms contain detailed information on administrative and clinical data. Main variables are shown in Table 1. At time of diagnosis, the department that verifies the diagnosis enters the first registration form (Registration Form), which must be entered within 6 months after diagnosis. The form contains information about the diagnosis and date of diagnosis, symptoms, and paraclinical, cytogenetic, and molecular profiles. The second registration form (the 2-Year Registration Form), entered 2 years after diagnosis, includes information on disease progression and the treatment initiated within the first 2 years. The third registration form (the 5-Year Registration Form), submitted 5 years after diagnosis, was introduced in 2015 and includes variables related to disease progression, cytogenetic and molecular assessment, and treatment. At end of followup (eg, death or progression to acute leukemia), a fourth registration form (the Follow-up Form), which includes information on date of last follow-up and vital status, must be completed.

\section{Follow-up}

\section{Validity and coverage}

The Danish Civil Registration System (CRS), ${ }^{4,5}$ with unique personal identification numbers that track each citizen nationwide, allows merging of data between different Danish registries, ${ }^{6,7}$ including the DCMR and the Danish National Patient Registry (DNPR). The DNPR contains information on all in- and outpatient hospital visits in Denmark, ${ }^{8,9}$ and the coding procedures for hematological 


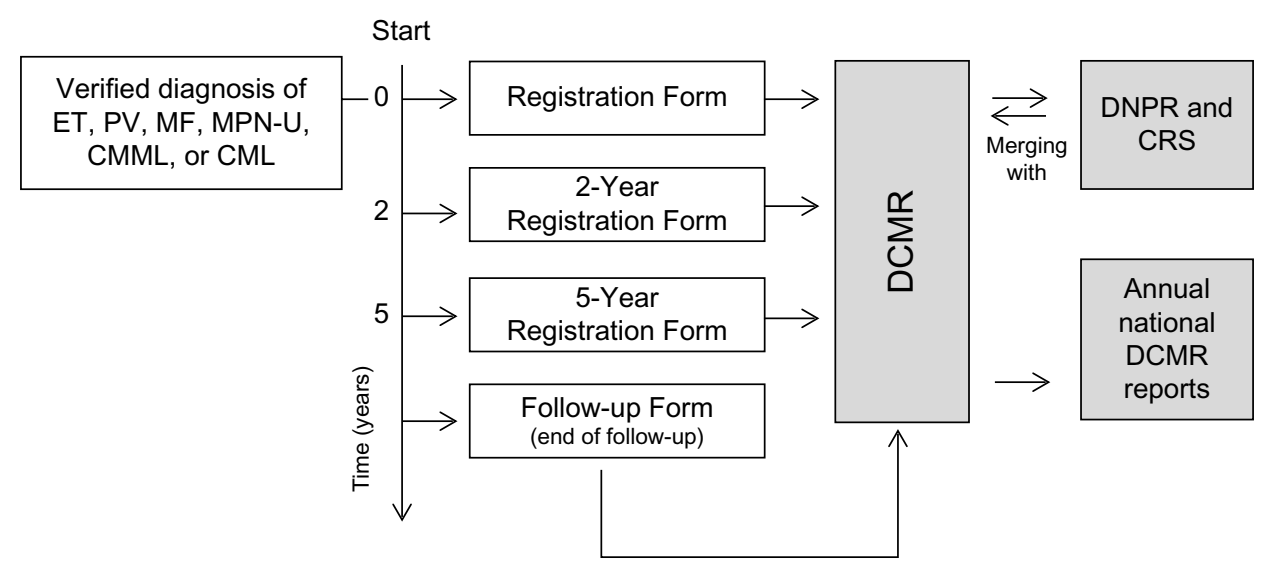

Figure I The Danish National Chronic Myeloid Neoplasia Registry algorithm.

Note: Data are entered at predefined intervals for each patient (at time of diagnosis, after 2-year and 5-year follow-ups, and at end of follow-up).

Abbreviations: CML, chronic myeloid leukemia; CMML, chronic myelomonocytic leukemia; CRS, The Danish Civil Registration System; DCMR, The Danish National Chronic Myeloid Neoplasia Registry; DNPR, The Danish National Patient Registry; ET, essential thrombocythemia; MF, myelofibrosis; MPN-U, unclassifiable myeloproliferative neoplasm; PV, polycythemia vera.

diseases show high validity. ${ }^{10,11}$ Because vital status and date of death are available for all citizens in the CRS, data from the DCMR are merged with the CRS using an administrative algorithm. Entering data on all patients in the database is mandatory and automatic cross-merging with the DNPR provides departments with follow-up lists (names and identification numbers), including a data list of correctly entered patients as well as patients whose data have not yet been correctly entered in the DCMR. This is done by extracting codes from the DNPR that correspond to chronic myeloid malignancies at any hematology department in Denmark. As a result, no patients are lost to follow-up in the DCMR. Furthermore, the registration system allows departments to change incorrectly entered data manually, resulting in subsequent data correction within the database, eg, if an incorrect diagnosis has been entered for a patient. The cross-merging of data between the DNPR and DCMR enables each department to maintain high data coverage, and it is an effective attempt to increase the validity of the DCMR.

\section{Annual reports}

The steering committee of the Danish National Chronic Myeloid Neoplasia Study Group (DCMSG) annually publishes an online report in Danish based on data from the DCMR. The report includes eight quality indicators that encompass database coverage, mortality, number of patients included in protocols, and cytogenetic and molecular biological assessment calculated for patients included within the past year. Data completeness (patients with submitted 2-Year Registration Forms) and long-term follow-up with survival estimates are also included, using data from preceding years. In addition, data are cross-merged with the DNPR to calculate coverage and completeness each year. The indicators assess quality of the database (coverage and completeness) as well as the quality of patient-related outcomes such as survival rates. The indicators are reported yearly at the departmental, regional, and national levels. National quality indicator results from the previous 3 years are shown in Table 2. In total, 2,690 patients were included in the DCMR from 2010 to 2014 (essential thrombocythemia $=744$, polycythemia vera $=729$, myelofibrosis $=374$, myeloproliferative neoplasms $=370$, chronic myelomonocytic leukemia $=196$, chronic myeloid leukemia =277), and consistently high national coverage ( $\geq 90 \%$ ) and completeness ( $\geq 88 \%$ ) have been observed since the registry was established. ${ }^{12}$

\section{Administrative issues and funding}

The DCMR is approved as a clinical quality database by the Danish authorities - the National Board of Health and the Danish Data Protection Agency. The Danish Common Hematological Database, including the DCMR, is part of the Danish Multidisciplinary Cancer Groups, which receive public financial support from the Danish regions. The Registry Support Centre of Epidemiology and Biostatistics (East) ensures administration of the database, support, and maintenance of the operating system and provides statistical analyses and epidemiological assistance for the annual reports, published in collaboration with the DCMSG. The academic and professional responsibility for maintenance and use of the DCMR lies within the DCMSG, (including the development and optimization of the database by 
Table I Main variables recorded on standardized online registration forms in the Danish National Chronic Myeloid Neoplasia Registry

\begin{tabular}{|c|c|c|}
\hline \multirow{2}{*}{$\begin{array}{l}\text { Registration form } \\
\text { (time of registration) }\end{array}$} & \multicolumn{2}{|l|}{ Main variables } \\
\hline & Nondisease-specific variables & Disease-specific variables \\
\hline \multirow{15}{*}{$\begin{array}{l}\text { Registration form } \\
\text { (submitted at time of } \\
\text { diagnosis) }\end{array}$} & Name and civil registration number & ET, PV, MF, MPN-U \\
\hline & Diagnosis according to WHO 2008/ICD-10 & Prior other cancer (solid/nonsolid) \\
\hline & Date of diagnosis & Inherited MPN predisposition \\
\hline & Prior hematological disease ( $>3$ months' duration) & Prior thrombosis (specified, if present) \\
\hline & B-symptoms: weight loss, night sweat, and itching & Bleedings \\
\hline & Spleen enlargement (if yes, then clinical or image & Musculoskeletal symptoms (related to MPN) \\
\hline & verified) & Microvascular symptoms (specified, if present) \\
\hline & Laboratory values & Cardiovascular risk factors \\
\hline & Biological material for a biobank & $\begin{array}{l}\text { Cytogenetic and molecular biological assessments: } \\
\text { JAK2V6I7F, JAK2 exon I2, MPL, CALR }, \text { ASXLI } \\
\text { If present, JAK2V6I7F, MPL, and CALR allele burden }{ }^{\mathrm{a}}\end{array}$ \\
\hline & & CML \\
\hline & & BCR-ABLI expression level (\% IS) ${ }^{\mathrm{a}}$ \\
\hline & & Chromosomal abnormalities, besides $\mathrm{t}(9 ; 22)$ \\
\hline & & Disease phase: chronic, accelerated, blast crisis \\
\hline & & CMML \\
\hline & & Cytogenetic and molecular biological assessment (r-IPSS) \\
\hline
\end{tabular}

2-Year Registration Form (submitted 2 years after diagnosis)

\section{Date of registration}

Spleen enlargement or progression ${ }^{\mathrm{a}}$

Cytogenetic and molecular biological assessment

Treatment (within the first 2 years)

Participation in clinical research protocol

Progression of disease
5-Year Registration Form (submitted 5 years after diagnosis)

\author{
Transplantation: date and type of transplantation \\ Date of registration \\ Spleen enlargement or progression ${ }^{\mathrm{a}}$ \\ Cytogenetic and molecular biological assessment \\ Treatment \\ Participation in clinical research protocol \\ Progression of disease \\ Transplantation: date and type of transplantation
}

\author{
ET, PV, MF, MPN-U \\ Vascular events since diagnosis ${ }^{\mathrm{a}}$ (specified if present) \\ If present, JAK2V6I7F, MPL, and CALR allele burden ${ }^{\mathrm{a}}$ \\ CML \\ Monitoring with $\mathrm{QPCR}$ or $\mathrm{dPCR}$ \\ BCR-ABLI expression level (\% IS) ${ }^{\mathrm{a}}$ \\ Chromosomal abnormalities besides $\mathrm{t}(9 ; 22)^{\mathrm{a}}$ \\ Mutation status in patients with treatment resistance ${ }^{a}$ \\ Treatment response $\mathrm{e}^{\mathrm{a}}$ \\ Vascular events since diagnosis ${ }^{\mathrm{a}}$ (specified if present) \\ ET, PV, MF, MPN-U \\ Vascular events since diagnosis ${ }^{a}$ (specified if present) \\ If present, JAK2V6I7F, MPL, and CALR allele burden ${ }^{\mathrm{a}}$ \\ CML \\ Monitoring with $\mathrm{QPCR}$ or $\mathrm{APCR}$ \\ BCR-ABLI expression level (\% IS) ${ }^{\mathrm{a}}$ \\ Chromosomal abnormalities besides $\mathrm{t}(9 ; 22)^{\mathrm{a}}$ \\ Mutation status in patients with treatment resistance ${ }^{a}$ \\ Treatment response ${ }^{a}$ \\ Vascular events since diagnosis ${ }^{\mathrm{a}}$ (specified if present)
}
Follow-up Form
Vital status
(submitted at end of
Date of follow-up or date of death
follow-up)
Place of death

Note: antroduced in 2015.

Abbreviations: ASXLI, the additional sex combs-like gene; BCR-ABLI, BCR-ABLI fusion gene (Philadelphia chromosome); CALR, calreticulin; CML, chronic myeloid leukemia; CMML, chronic myelomonocytic leukemia; APCR, digital polymerase chain reaction; ET, essential thrombocythemia; ICD, International Classification of Diseases; IS, International Scale; JAK2V6I7F, janus kinase2V6I7F mutation; MF, myelofibrosis; MPL, myeloproliferative leukemia protein (thrombopoietin receptor); MPN, myeloproliferative neoplasm; MPN-U, unclassifiable myeloproliferative neoplasms; PV, polycythemia vera; qPCR, quantitative polymerase chain reaction; WHO, World Health Organization.

improving registration forms, introducing new variables, and surveying variables). Access to data from the DCMR can be obtained by applying to the Registry Support Centre of Epidemiology and Biostatistics, but researchers can only be granted access after approval from the Danish authorities.

\section{Conclusion}

Hematology departments in Denmark enter data into the DCMR through the online registration system, to generate information on a nationwide cohort of patients with chronic myeloid malignancies. The DCMR ensures that departments have the possibility of monitoring diagnosis and treatment 
Table 2 Quality indicators in the Danish National Chronic Myeloid Neoplasia Registry

\begin{tabular}{|c|c|c|c|}
\hline \multicolumn{4}{|c|}{ National quality indicators in the DCMR (\%) } \\
\hline Year & 2014 & 2013 & 2012 \\
\hline \multicolumn{4}{|c|}{ Database-related indicators (quality of the database) } \\
\hline Database coverage ${ }^{a}$ & 90 & 97 & 98 \\
\hline $\begin{array}{l}\text { Data completeness (submitted } \\
\text { 2-Year Registration Forms) }\end{array}$ & 88 & 96 & 99 \\
\hline \multicolumn{4}{|c|}{ Patient-related indicators (quality of patient-related outcomes) } \\
\hline 30-day mortality & 0.5 & 0.5 & 0.6 \\
\hline 180-day mortality & 3.7 & 3.9 & 4.5 \\
\hline $\begin{array}{l}\text { Cytogenetic or molecular biological } \\
\text { diagnostics performed }\end{array}$ & 97 & 97 & 98 \\
\hline Patients included in a clinical protocol & 14 & 2 & 3 \\
\hline Year of registration & $2013-2014^{b}$ & $2011-2012^{b}$ & \\
\hline \multirow[t]{2}{*}{ I-year survival } & 92 & 92 & - \\
\hline & $20 I I-2014^{b}$ & - & - \\
\hline 3-year survival & 80 & - & - \\
\hline
\end{tabular}

Notes: National quality indicator results (calculated for the years 2012-2014). Each indicator is calculated as percentage of patients that meet the indicator criteria.

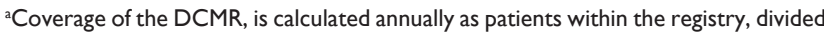
by all patients with chronic myeloid malignancies in hematology departments in Denmark. 'Patients with registration in the database over the years are pooled. Abbreviation: DCMR, Danish National Chronic Myeloid Neoplasia Registry.

in patients at predefined intervals and comparing quality indicator results with national levels yearly. Recent quality studies of Danish hematological databases, the Danish National Lymphoma Registry, and the Danish National Acute Leukemia Registry, which use the same reporting tool and trained health care professionals for entering data, show high data quality. ${ }^{13,14}$ However, generated data in the DCMR must be used with caution, until a validity study has been undertaken and data quality has been assessed. The DCMR, a population-based clinical quality database (with high coverage), represents a unique data source for physicians to scrutinize and monitor the clinical quality of diagnosis and treatment of Danish patients with chronic myeloid malignancies. In addition, the database addresses research-related questions, generating valuable information of interest to clinicians and researchers, as well as patients and politicians.

\section{Acknowledgments}

The authors thank all members of the DCMSG, each hematology department, and staff for their continuous commitment to managing patients with chronic myeloid malignancies and collecting data. In addition, we thank Dr Peter de
Nully Brown for valuable advice on the management and optimization of the DCMR. This paper was funded by the Program for Clinical Research Infrastructure, established by the Lundbeck Foundation and the Novo Nordisk Foundation and administered by the Danish Regions. The Danish Cancer Society also supported this work.

\section{Disclosure}

The authors report no conflicts of interest in this work.

\section{References}

1. Tefferi A, Vardiman JW. Classification and diagnosis of myeloproliferative neoplasms: the 2008 World Health Organization criteria and point-of-care diagnostic algorithms. Leukemia. 2008;22:14-22.

2. Statistics Denmark [webpage on the Internet]. Population and Elections. Available from: http://www.statbank.dk/statbank5a/default.asp?w=1536. Accessed July 9, 2015.

3. The Danish Health and Medicine Authority [webpage on the Internet]. Pakkeforløb for hæmatologiske Kræftformer Hematological Cancer Care Packages] 2012:06. Version 3.0. Danish. Available from: http:// sundhedsstyrelsen.dk/da/udgivelser/2012/pakkeforloeb-for-kroniskemyeloide-sygdomme. Accessed July 9, 2015.

4. Pedersen CB. The Danish Civil Registration System. Scand J Public Health. 2011;39(7 suppl):22-25.

5. Schmidt M, Pedersen L, Sørensen HT. The Danish Civil Registration System as a tool in epidemiology. Eur J Epidemiol. 2014;29(8): 541-549.

6. Frank L. Epidemiology: when an entire country is a cohort. Science. 2000;287(5462):2398-2399.

7. Green A. Danish clinical databases: an overview. Scand J Public Health 2011;39(7 suppl):68-71.

8. Schmidt M, Schmidt SAJ, Sandegaard JL, Eherenstein V, Pedersen L, Sørensen HT. The Danish National Patient Registry: a review of content, data quality, and research potential. Clin Epidemiol. 2015;7: 449-490.

9. Andersen TF, Madsen M, Jørgensen J, Mellemkjoer L, Olsen JH. The Danish National Patient Register. A valuable source of data for modern health sciences. Dan Med Bull. 1999;46(3):263-268.

10. Nickelsen TN. Data validity and coverage in the Danish National Health Registry. A literature review. Dan Med Bull. 2001;164(1):33-37.

11. Nørgaard M, Skriver MV, Gregersen H, Pedersen G, Schønheyder HC, Sørensen HT. The data quality of haematological malignancy ICD-10 diagnoses in a population-based hospital discharge registry. Eur J Cancer Prev. 2005;14(3):201-206.

12. Danish National Chronic Myeloid Neoplasia Study Group [homepage on the Internet]. DCMSG. Årsrapport. [Annual Chronic Myeloid Neoplasia Report]; 2014. Danish. Available from: http://www.myeloid. $\mathrm{dk} /$. Accessed December 28, 2015.

13. Ostgård LSG, Nørgaard JM, Severinsen MT, et al. Data quality in the Danish National Acute Leukemia Registry: a hematological data resource. Clin Epidemiol. 2013;5:335-344.

14. Arboe B, Galaly TCE, Clausen MR, et al. The Danish Lymphoma Registry has a high coverage and high data quality. EHA Learning Center. Poster presented at: Annual Meeting of the European Hematology Association; June 12, 2015; 100652; Vienna, Austria. 


\section{Publish your work in this journal}

Clinical Epidemiology is an international, peer-reviewed, open access, online journal focusing on disease and drug epidemiology, identification of risk factors and screening procedures to develop optimal preventative initiatives and programs. Specific topics include: diagnosis, prognosis, treatment, screening, prevention, risk factor modification,

Submit your manuscript here: http://www.dovepress.com/clinical-epidemiology-journal systematic reviews, risk \& safety of medical interventions, epidemiology \& biostatistical methods, and evaluation of guidelines, translational medicine, health policies \& economic evaluations. The manuscript management system is completely online and includes a very quick and fair peer-review system, which is all easy to use. 